\title{
Determining the orientation of depth-rotated familiar objects
}

\author{
RyosuKe NiIMI AND KaZUhiko Yokosawa \\ University of Tokyo, Tokyo, Japan
}

\begin{abstract}
How does the human visual system determine the depth-orientation of familiar objects? We examined reaction times and errors in the detection of $15^{\circ}$ differences in the depth orientations of two simultaneously presented familiar objects, which were the same objects (Experiment 1) or different objects (Experiment 2). Detection of orientation differences was best for $0^{\circ}$ (front) and $180^{\circ}$ (back), while $45^{\circ}$ and $135^{\circ}$ yielded poorer results, and $90^{\circ}$ (side) showed intermediate results, suggesting that the visual system is tuned for front, side and back orientations. We further found that those advantages are due to orientation-specific features such as horizontal linear contours and symmetry, since the $90^{\circ}$ advantage was absent for objects with curvilinear contours, and asymmetric object diminished the $0^{\circ}$ and $180^{\circ}$ advantages. We conclude that the efficiency of visually determining object orientation is highly orientation-dependent, and object orientation may be perceived in favor of front-back axes.
\end{abstract}

The human visual system maintains object constancy in recognizing familiar objects despite changes in orientation and the resultant variations in appearance. However, it is also important to detect changes in the orientation of objects. Information about object orientation logically enables transformations between object-centered reference frames and other reference frames. This contributes to activities such as the manipulation of tools and visual perception of natural scenes. Recent neurophysiological evidence from monkeys suggests that the orientation coding of faces occurs in the object-recognition-related brain area (Wang, Tanifuji, \& Tanaka, 1998) whereas 3-D orientation coding of simple geometric objects occurs in the hand-manipulation-related area of the monkey brain (for review, see Sakata et al., 1999).

The present study examined human visual determination of the orientation of depth-rotated familiar objects relative to the observer. Since detecting orientation change involves detecting changes of object appearance, it is difficult to detect any orientation change that produces slight or no changes in object appearance. For instance, a smooth uniformly colored vertical column produces no appearance changes when it rotates about its vertical axis, while it produces obvious change of appearance when it rotates about its horizontal axis. Tarr and Kriegman (2001) showed that human sensitivity to depth-orientation changes of geometric 3-D objects was high when orientation changes involved qualitative changes of appearances (e.g., emergence of new side of an object, disappearance of a T-junction of object contour). In other words, orientation changes from some particular orientations are visually salient while orientation changes from other orientations are not. This finding is consistent with the idea that an infinite number of appearances of a single object can be organized into a limited number of categories, or "views," that are qualitatively distinct from each other (Callahan \& Weiss, 1985; Koenderink \& van Doorn, 1979). Furthermore, Tarr (1995) suggested that such organization of multiple views is represented in the human visual object recognition mechanism.

How about familiar objects? Tarr and Kriegman (2001) used only two simple objects. Since familiar objects have more complex and varied shapes, it is likely that detecting orientation changes of familiar objects involves some other visual information specific to familiar objects. The torus and bell-shaped objects of Tarr and Kriegman were rotationally symmetric around their vertical axes and comprised of curvilinear contours, but many familiar objects are bilaterally symmetric and have linear, straight contours. In addition, familiar objects frequently have special orientations such as "front." If human vision is tuned for familiar objects, then a high sensitivity to orientation changes from their special orientations is expected.

In this paper we examined the effect of object orientation $\left(0^{\circ}=\right.$ front; $45^{\circ} ; 90^{\circ}=$ side; $135^{\circ} ; 180^{\circ}=$ back $)$ on the sensitivity to relative differences $\left(15^{\circ}\right)$ in the objects' orientations. We used familiar objects with salient topbottom and front-back axes. Participants observed two simultaneously presented pictures of objects and made judgments about whether they differed in orientation (present in half of the trials; see Figure 1B). We expected that performance speed and accuracy would be influenced by the objects' orientations. We further investigated the

R. Niimi, niimi@1.u-tokyo.ac.jp 
A
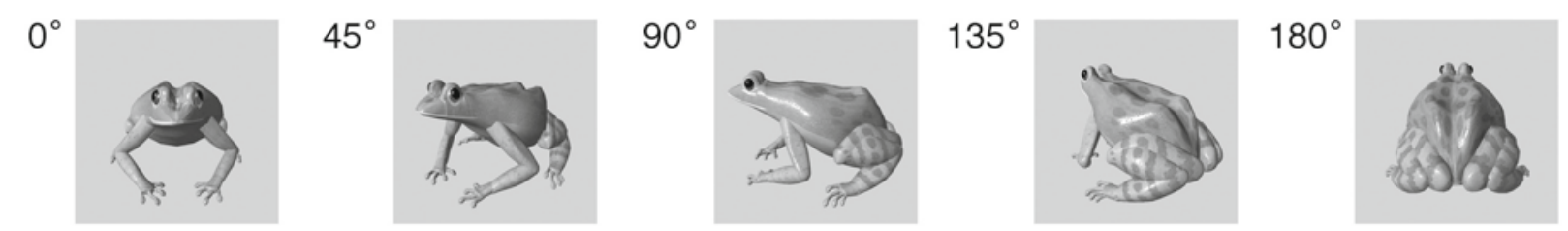

\section{Experiment 1}

B Different Trial
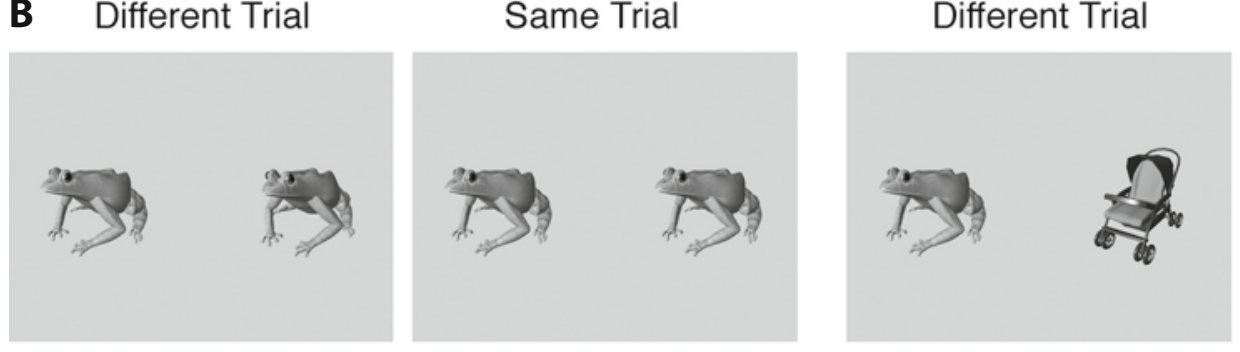

Experiment 2

C

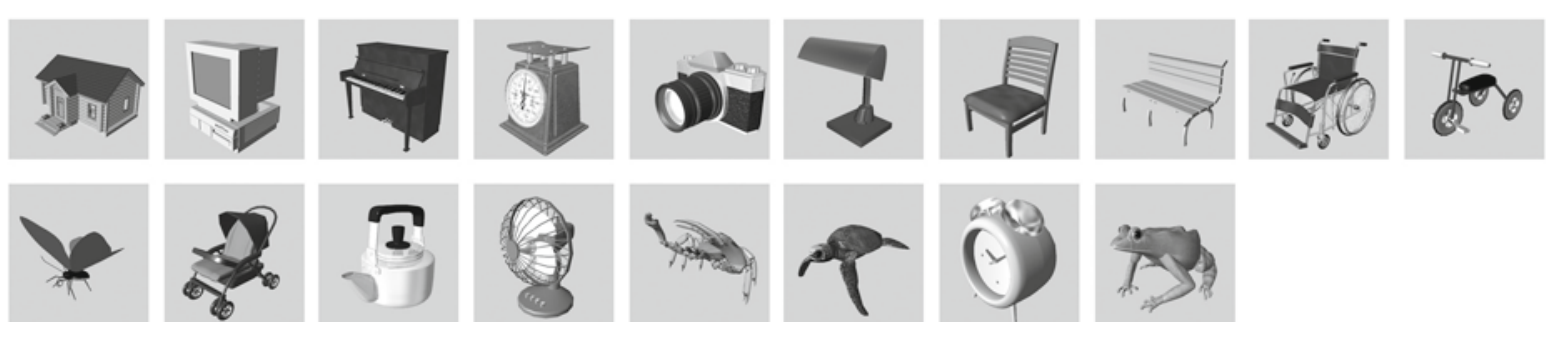

Figure 1. The five standard orientations (A), and samples of stimuli displays (B). The same-trial-object pair matched in their orientations (one of the standard orientations; the figure shows $45^{\circ}$ as an example), whereas the different-trial pair differed in their orientations (one of the standard orientations and a $15^{\circ}$ deviation from it, $45^{\circ}$ and $30^{\circ}$ for example). (C) The 18 familiar objects adopted as experimental stimuli (shown in $45^{\circ}$ orientation). The upper 10 objects were linear objects and the lower 8 objects were curvilinear objects, shown in the order of rated linearity scores (see text for details).

contributions of visual information, such as linear contour and symmetric shape of the object, to the observed performances.

\section{EXPERIMENT 1}

\section{Method}

Participants. Twelve individuals ( 8 females and 4 males, 19-25 years of age) participated. They all had normal or correctedto-normal vision.

Stimuli. We chose 24 familiar objects (18 for the experimental session, 6 for the practice session) from commercial 3-D model data sets. All objects were bilaterally symmetric. We avoided elongated or planar objects because their orientation changes yield radical changes in aspect ratios in 2-D views. Our 24 objects had no more than a 1.7 ratio among width, height and depth. We equalized the numbers of width-longest, height-longest and depth-longest objects $(6,6$, and 6 objects, respectively, then 18 experimental objects). The objects were rendered into colored stimuli pictures with various orientations that were rotations about the vertical axis $(-15$ to $+195^{\circ}$ — see below for details). The viewpoints were $20^{\circ}$ elevated from the horizontal plane.

Design and Procedure. Each trial started with an $800 \mathrm{msec}$ fixation cross, followed by the simultaneous presentation of two picture stimuli of identical objects. One stimulus was one of the standard stimuli (see below). The other was a picture in which the object orien- tation deviated $\pm 15^{\circ}$ from the standard stimulus (different trials, $50 \%$ of the trials) or the identical picture (same trials, $50 \%$ of the trials). The object orientations (azimuth angles) were $0^{\circ}, 45^{\circ}, 90^{\circ}, 135^{\circ}$, and $180^{\circ}$ from the standard stimuli (Figure 1A). Participants were not informed about these orientations. We asked 15 individuals (not participants in the experiments) to choose the "front view" from the standard stimuli of each object. The proportion choosing the $0^{\circ}$ view as the front was $95.6 \%$, which confirms that the objects have salient front-back axes. The task was to decide whether there was a difference in object orientation between the two stimuli and press the appropriate response key for different orientation or same orientation as quickly and accurately as possible. The stimuli were presented until a response was made or $5,000 \mathrm{msec}$ passed. No feedback on accuracy was given.

Stimuli were presented at the center of the 21 -in. color CRT screen (refresh rate $75 \mathrm{~Hz}$ ). Participants sat $80 \mathrm{~cm}$ away from the screen, with their heads fixed in a chinrest. Each object picture stimuli subtended a maximum visual angle of $7.3^{\circ} \times 7.3^{\circ}$, with the centers of the two simultaneously presented pictures separated horizontally by $11.0^{\circ}$. The background was uniformly gray.

Each participant performed practice and experimental sessions. They read a list of the names of the upcoming objects before each session, to minimize any cost of object identification. The six objects in the practice session never appeared in the experimental session. The practice session ( 24 trials) was repeated until the error rate fell below $20 \%$. The experimental session consisted of 360 trials in which all conditions were counterbalanced and randomly ordered ( 5 orientations of standard stimuli $\times$ different or same $\times$ orientation difference 
was $+15^{\circ}$ or $-15^{\circ} \times 18$ objects). Self-timed breaks were introduced after every 12 trials. This experiment took approximately $35 \mathrm{~min}$.

\section{Results and Discussion}

We considered trials with an extremely fast response $(<200 \mathrm{msec})$ or no response as outliers, but there were no such trials. The effects of object orientation $\left(0^{\circ}, 45^{\circ}, 90^{\circ}\right.$, $\left.135^{\circ}, 180^{\circ}\right)$ and trial type (different or same) were analyzed using a two-way repeated measures ANOVA. The mean RTs on correct-response trials (Figure 2A) showed significant main effects for both object orientation and trial type $[F(4,44)=44.23, p<.01 ; F(1,11)=8.83, p<$ .05 , respectively]. The interaction was also significant $[F(4,44)=5.65, p<.01]$. The simple main effect of object orientation was significant on both trial types $(p<$ .01 ). Post hoc analyses (Tukey's HSD) revealed that $0^{\circ}$ and $180^{\circ}$ produced faster responses than $45^{\circ}, 90^{\circ}$ and $135^{\circ}$. Regarding the interaction, RTs for $90^{\circ}$ also were faster in different trials but not in same trials. The ANOVA for the error rate data showed a similar effect of object orientation. The main effect of object orientation and the interaction were significant $[F(4,44)=9.88, p<.01 ; F(4,44)=$ $4.35, p<.01$, respectively], while the main effect of trial type was not $(F<1)$. The simple main effect of object orientation was significant only in the different trials ( $p<$ .01 ), and post hoc analyses (Tukey's HSD) again revealed the $0^{\circ}$ and $180^{\circ}$ advantages. These results show that efficiency of detecting differences of objects' depth orientation is orientation dependent. Human vision is accurate in detecting deviation from direct front and back orientations, and relatively inaccurate in detecting deviation from oblique orientations, with the results being in between for side views $\left(90^{\circ}\right)$. The same ANOVAs on data averaged over objects rather than participants showed a similar pattern of results.

What kind of visual features contributed to the results? According to the participants' introspective reports, they tried to utilize (1) differences in color and brightness that were due to differences in shading and glossy highlights, (2) spatial relationships of parts (e.g., whether the frog's right leg occluded the left leg), (3) obliqueness of linear contour, (4) closed contour shape changes, and (5) (dis)appearance of parallelepiped-like objects' faces (e.g., whether the facade of the house is visible). The use of (2) and (5) is consistent with the discussion of Tarr and Kriegman (2001). Local visual differences (1) seem useful only for detecting orientation differences of identical objects. Their possible contribution was tested further in Experiment 2. Obliqueness of linear contour (3) might be useful for determining orientation of familiar objects that have linear contours, and could contribute to the better performances for the $0^{\circ}, 90^{\circ}$, and $180^{\circ}$ orientations, because our stimuli included constructed objects that frequently produced exact horizontal linear contours in those cardinal orientations. Closed contour shape changes (4) do not seem as relevant to the orientation dependence of the performances, except for symmetry of object contours. We tested the effect of contour symmetry further (see below), although no participant explicitly referred to using symmetry in making judgments.

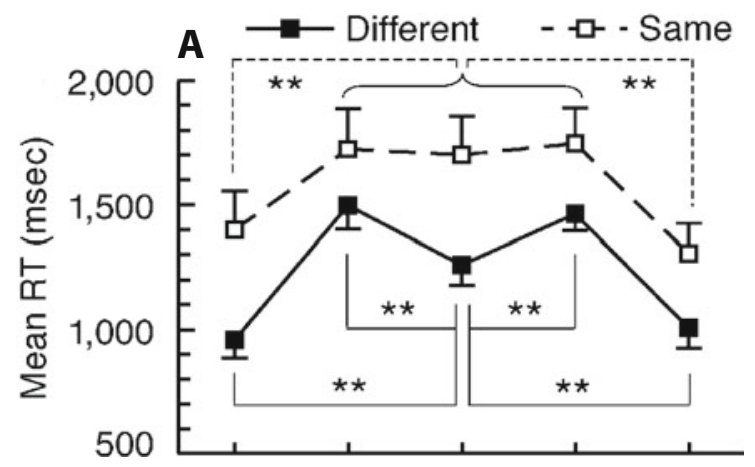

B
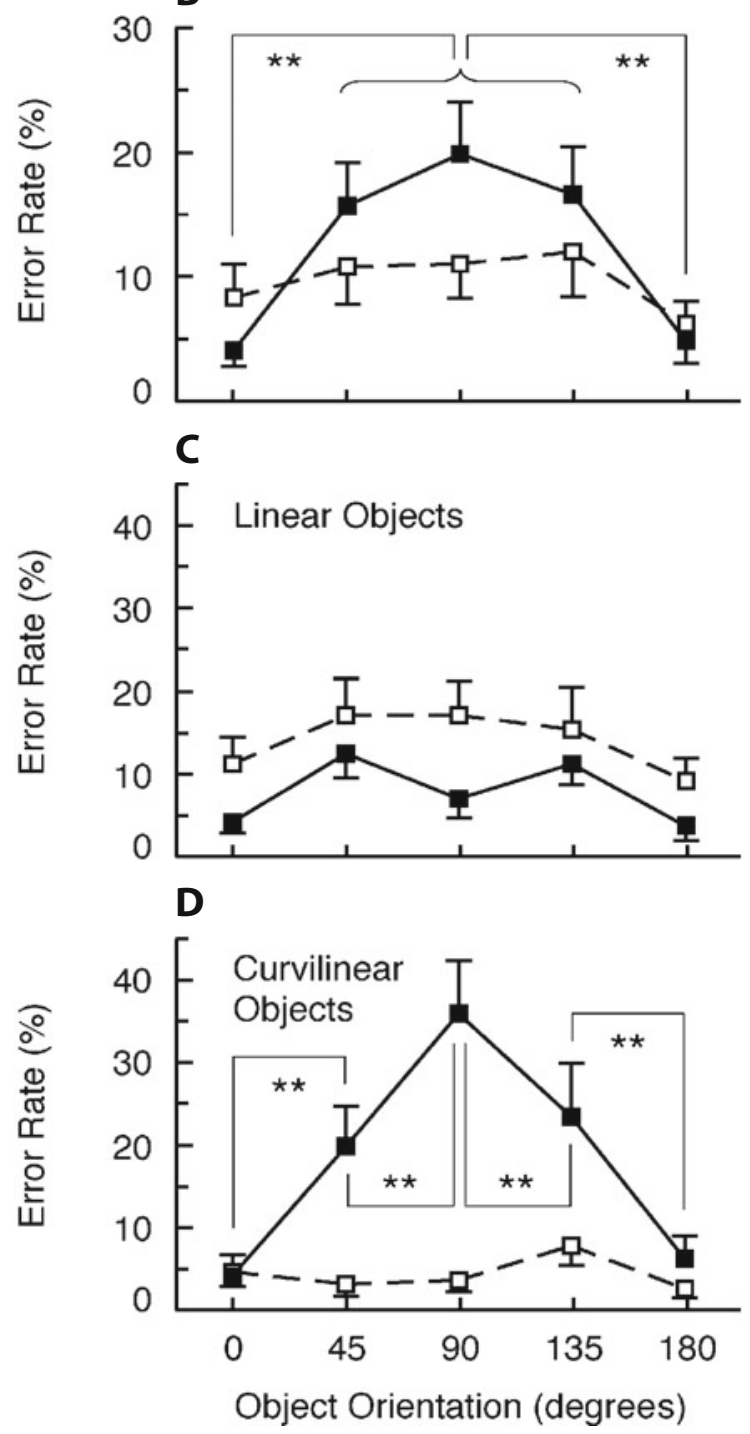

Figure 2. Results of Experiment 1. Mean RT (A) and mean error rate $(B)$ for the orientation difference detection task as a function of the standard orientations. In addition, trials with curvilinear contour objects and trials with linear contour objects showed a different tendency in error rates (C and D; see text for details). Error bars represent standard errors. Results of post hoc comparisons (Tukey's HSD) of the simple main effects of object orientation are schematically shown (solid lines for comparisons among different trials, dashed lines for comparisons among same trials). ${ }^{* *} p<.01$. 
The effect of linear contours. Since familiar constructed objects often are made with linear contours that are parallel or orthogonal to their front-back axes, horizontal edges are frequently present in their direct front, back and side views. Such edges immediately deviate from horizontal orientation when the objects are depth-rotated. We examined whether linear contours can explain the advantages of the $0^{\circ}, 90^{\circ}$, and $180^{\circ}$ orientations found in Experiment 1.

First, we assessed the contour linearity of the 18 objects used in the experimental sessions. Twelve individuals (who participated in neither Experiment 1 nor Experiment 2) were given a booklet in which all the standard stimuli were printed, and instructed to rate "how much does the contour of the object contain straight lines" for every object. Their answers were made by marking on rating scales that ranged from 1 to 7 . The 10 objects with averaged scores more than 4 were classified as linear objects, and the other 8 objects were classified as curvilinear objects (Figure 1C). We reanalyzed the Experiment 1 data according to this classification. While the two categories replicated the $0^{\circ}, 90^{\circ}$, and $180^{\circ}$ advantages for RT results, they differed in error rate results (Figures 2C and 2D). For linear objects, the main effect of object orientation was significant $[F(4,44)=3.67, p<$ $.05]$, but not the interaction. Tukey's HSD post hoc analyses of the main effect revealed more errors for $45^{\circ}$ than for $180^{\circ}$ $(p<.05)$. For curvilinear objects, we found a significant main effect of object orientation $[F(4,44)=12.93, p<$ $.01]$ and the interaction was significant $[F(4,44)=13.33$, $p<.01]$. The simple main effect of object orientation was significant only for different trials $(p<.01)$, and post hoc analyses (Tukey's HSD) revealed that error rates were higher for $90^{\circ}$ and lower for $0^{\circ}$ and $180^{\circ}$. Taken together, we found a disadvantage at $90^{\circ}$ for curvilinear objects, which was not found for linear objects, where the linear contours improved the accuracy of determining the $90^{\circ}$ orientations. However, the $0^{\circ}$ and $180^{\circ}$ advantages were observed in RT and error rate results irrespective of contour linearity.

This analysis suggests that linear contour is a visual cue for object orientation. The strong bias toward "same" responses for curvilinear objects (Figure 2D) further supports the finding; participants might overlook the differences in orientation of curvilinear objects because they could not find orientation differences of linear contours. The orientation spectrum of Fourier transformations of edge-enhanced stimulus images indicated that horizontal edges were dominant in the $0^{\circ}, 90^{\circ}$, and $180^{\circ}$ images of linear objects but not in those images of curvilinear objects. A high precision of human vision to horizontal orientation rather than to oblique orientations (oblique effect; Heeley \& Timney, 1988) might contribute to determination of an exact $90^{\circ}$ orientation. It may also contribute to determination of the $0^{\circ}$ and $180^{\circ}$ orientations, but we did not find a linear-object advantage at those orientations, probably because of a ceiling effect. Since the $0^{\circ}$ and $180^{\circ}$ advantages were found even for curvilinear objects, these advantages are not fully attributable to linear contours.

The effect of symmetry. Since our stimuli objects were symmetric about their midsagittal plane, their $0^{\circ}$ and $180^{\circ}$ views had symmetric contours (Figure 1A). 2-D symmetry is a salient visual feature (Barlow \& Reeves, 1979;
Wagemans, 1995), and a cue for front-back orientations of objects (McBeath, Schiano, \& Tversky, 1997; Zabrodsky, Peleg, \& Avnir, 1995). Orientation changes from exact front or back cause degradation of symmetry, and might improve the $0^{\circ}$ and $180^{\circ}$ performances. The results of our supplementary experiment supported this interpretation. Ten individuals performed the same experimental task with 10 asymmetric stimuli objects. ${ }^{1}$ For the RT results (Figure $3 \mathrm{~A}$ ), post hoc analyses showed that in Different trials, $0^{\circ}, 90^{\circ}$, and $180^{\circ} \mathrm{RTs}$ were significantly faster than $45^{\circ}$ and $135^{\circ}$ RTs. For the Same trials the RTs showed similar results. Because the front and back views were asymmetric, the $0^{\circ}$ and $180^{\circ}$ advantages over $90^{\circ}$ largely disappeared. The error rate results were consistent with the RT results but were not as statistically significant (Figure 3B).

\section{EXPERIMENT 2}

The Experiment 1 task could be performed by detecting local visual differences between the two stimuli (e.g., changes in shadings and highlights) rather than by deter-
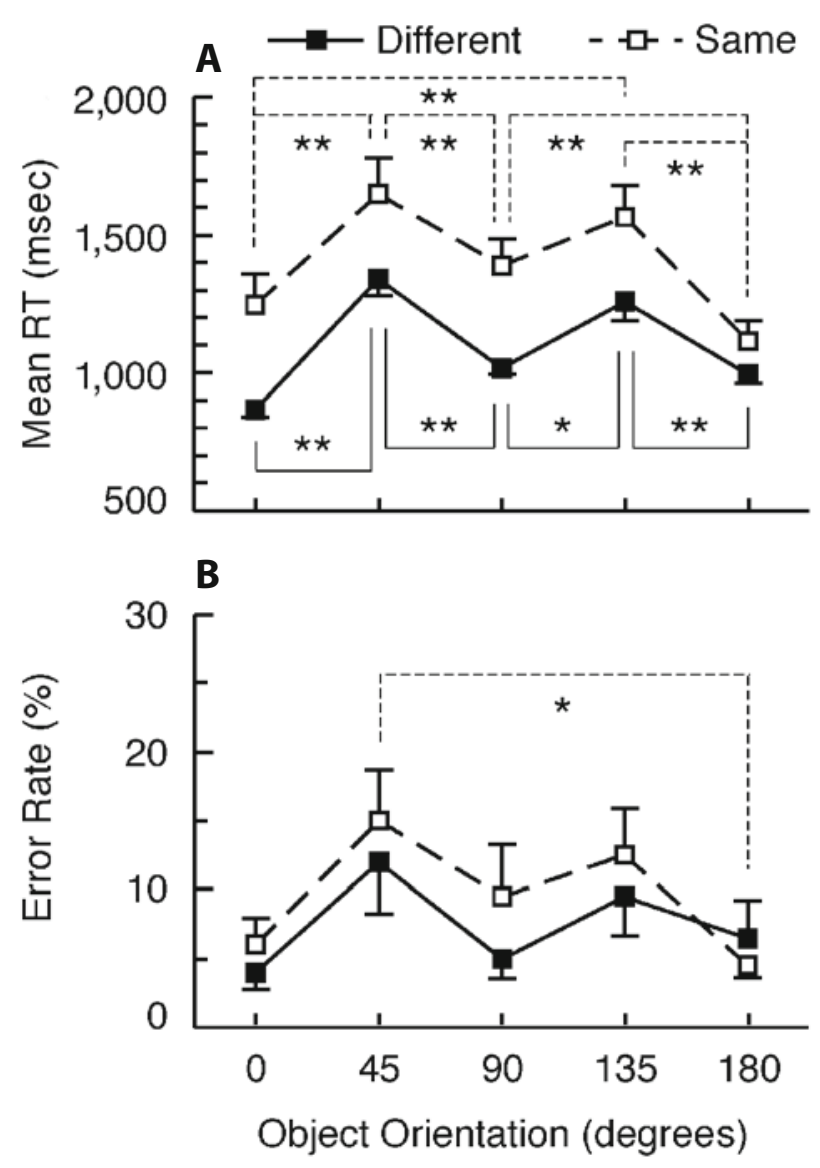

Figure 3. Results of the supplementary experiment that adopted asymmetric objects as stimuli. Mean RT (A), and mean error rate (B) of the orientation difference detection task as a function of the standard orientations. Error bars represent standard errors. Results of post hoc comparisons (Tukey's HSD) on the simple main effects of object orientation were schematically shown (solid lines for comparisons among different trials, dashed lines for comparisons among same trials). ${ }^{*} p<.05 .{ }^{* *} p<.01$. 
mining object orientations. To examine the possible contribution of local differences, Experiment 2 investigated the detection of orientation differences between objects with distinct identities. Picture stimuli of two different objects were simultaneously presented, and participants were required to detect $15^{\circ}$ orientation differences (Figure 1B). There was no benefit from detecting local differences for this task. We predicted that the $0^{\circ}, 90^{\circ}$, and $180^{\circ}$ advantages would remain evident because local differences seem unlike to be more salient in those orientations.

\section{Method \\ Participants. Twelve individuals ( 7 females and 5 males, 21-26 years of age) participated. They all had normal or corrected- to-normal vision, and had not participated in Experiment 1. \\ Design and Procedure. All the stimulus pictures, task and ap- paratus were identical to Experiment 1. However, in this experiment a standard stimulus and a stimulus of a different object in a $15^{\circ}$ de- viated orientation (different trial) or in the same orientation (same trial) were presented simultaneously in every trial (Figure 1B). The pairings of objects were determined randomly and counterbalanced, with the constraint that any paired objects had a common longest axis (width, height, or depth). This constraint ensured that the Ex- periment 2 results would not be attributable to possible difficulty in comparing orientations of differently elongated objects. The other procedures were identical to Experiment 1.}

\section{Results and Discussion}

The results are graphed in Figure 4, and the same analyses as Experiment 1 were conducted. The ANOVAs for RTs were significant only for the main effect of object orientation $[F(4,44)=10.53, p<.01]$. We observed the faster RTs for $0^{\circ}$ and $180^{\circ}$ than for $45^{\circ}$ and $135^{\circ}$ (Figure $4 \mathrm{~A}$ ). Error rate results showed a significant main effect of object orientation and trial type $[F(4,44)=92.50, p<.01$; $F(1,11)=7.42, p<.05$, respectively], and a significant interaction $[F(4,44)=7.30, p<.01]$. This interaction seems to reflect the participants' bias for correct rejection (correct response in the same trials) rather than hit (correct response in different trials) only in the $90^{\circ}$ and $135^{\circ}$ conditions. The simple main effect of object orientation was significant for both trial types $(p<.01)$. As Figure 4B shows, we again observed the fewer errors for $0^{\circ}$ and $180^{\circ}$. In addition, the same trials produced fewer errors for $90^{\circ}$. To summarize, the $0^{\circ}$ and $180^{\circ}$ advantages were replicated in the RT and error rate results. The $90^{\circ}$ advantage was replicated too, but the effect seems relatively small.

Although the overall error rates were higher than in Experiment 1, suggesting that local differences partly improved performance in Experiment 1, the qualitative effect of object orientation remained the same. Thus the high sensitivities to object orientation for $0^{\circ}$ and $180^{\circ}$ (and even $90^{\circ}$ ) are not due to local differences. Furthermore, in Experiment 2 the different and same trials resulted in comparable RTs, whereas the different trial RTs were faster than the same trial RTs in Experiment 1. This change might reflect a tendency to search for local differences in Experiment 1; participants could respond immediately after any single difference was found in the different trials, whereas they might need a longer time to be sure that there was not any local difference in the same trials.
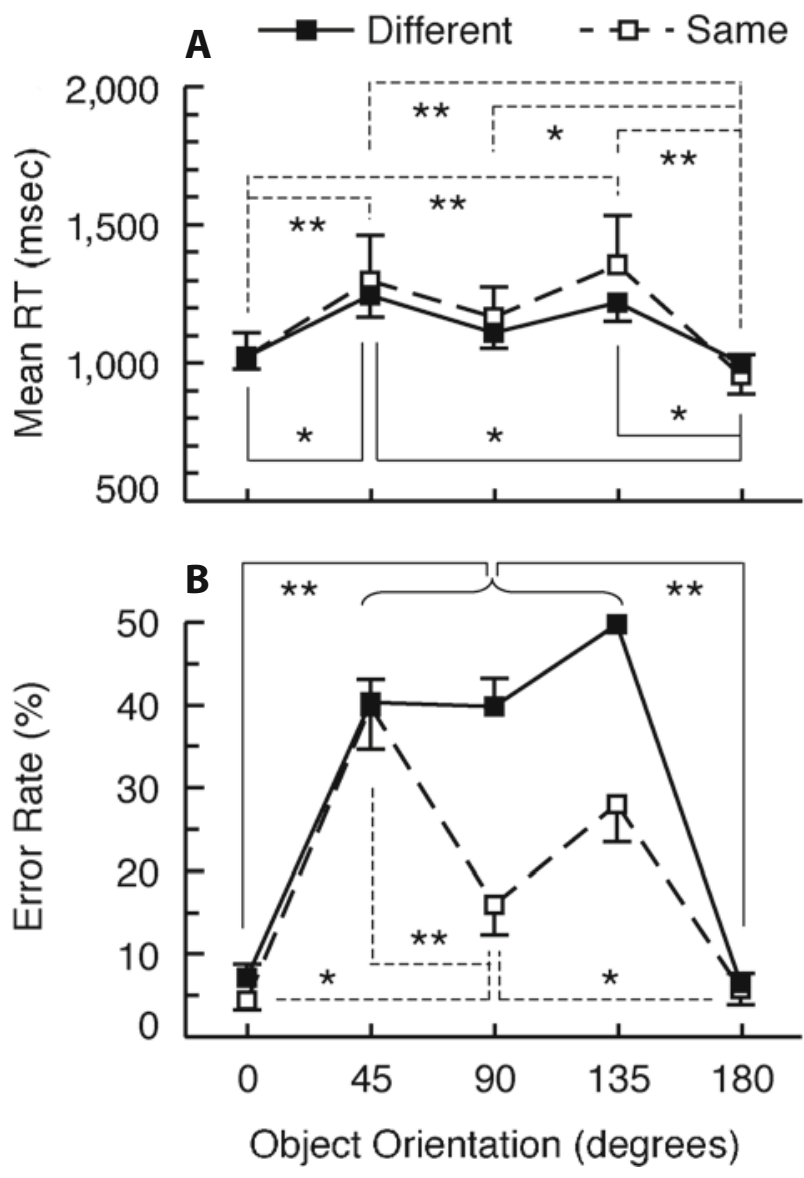

Figure 4. Results of Experiment 2 (orientation difference detection between distinctive objects). Mean RT (A) and mean error rate (B) as a function of the standard orientations. Error bars represent standard errors. Results of post hoc comparisons (Tukey's HSD) on the simple main effects of object orientation were schematically shown (solid lines for comparisons among different trials, dashed lines for comparisons among same trials). $\quad{ }^{*} p<.05 . \quad{ }^{* *} p<.01$.

\section{GENERAL DISCUSSION}

We found that the visual determination of the depth orientation of familiar objects is highly orientation dependent (relatively accurate for front, side and back orientations, but inaccurate for oblique orientations). The best performance was observed for front and back orientations, which suggests that human vision may have a good ability to determine front-back axes of objects. The performance was strongly influenced by the visual features of the stimulus objects, such as linear contours (Figures 2C and 2D). Besides the previous research using geometric objects (Tarr \& Kriegman, 2001), we suggest that horizontal linear contours and visual symmetry are utilized for determining the orientation of familiar objects. Horizontal contours may contribute to determining front, side and back orientations of many constructed objects that possess linear contours. Symmetric contour is a cue for front and back orientations, as nearly all symmetric familiar objects are symmetric about a midsagittal plane, not about a hori- 
zontal plane. Although it seems plausible that human vision is sensitive to front orientation because frontal views of objects seem more familiar, we found that this was not the case. We conducted an additional paper-and-pencil experiment asking new participants to estimate the view familiarity of the five standard orientations we used. The results for estimated familiarity did not correspond to the main experiment's results for orientation performance. Participants rated the $180^{\circ}$ (back) views, which had yielded superior performance, as least familiar, and rated the $45^{\circ}$ views, which had yielded poor performance, as the most familiar. Overall, front and back orientations of familiar objects provide orientation-specific features such as horizontal contours and symmetry, while oblique orientations do not. Thus changes from front and back (and side) orientations yield qualitative changes of object appearances, while changes from oblique orientations yield only metric changes in appearances. Human vision may categorize the orientations of familiar objects into front, back, side, and other oblique orientations. Because these effects seem to originate largely from image features (not view familiarity), equivalent effects may be observed even when novel objects with symmetry and linear contours are tested.

There are some other factors that may influence the processes of determining object orientation. One could be a depth cue such as binocular disparity or texture gradients on the horizontal ground, which were absent in our stimuli. An object viewed at a single azimuth angle can be differently projected onto the retinal image depending on the viewing distance and the elevation angle of the viewpoint. This uncertainty possibly contributes to the poorer determination of oblique orientations, and thus depth cues may partly compensate for such a disadvantage. Another untested factor is the axis of elongation of objects (current study excluded objects with salient axis of elongation as stimuli). Changes from an orientation in which the axis of elongation is foreshortened (e.g., a frontal view of horse) will cause radical changes in the appearance of the object, which could yield a high performance for that orientation.

The relationship between identification and orientation determination is important to understand. One may predict that determining orientation contributes to better constancy of object identification. However the known view-dependent efficiency of object identification has an opposite tendency to the observed efficiency of object orientation. The efficiency of identification is generally low for the front view but high for moderately oblique views (Lawson, Humphreys, \& Jolicœur, 2000; Mitsumatsu \& Yokosawa, 2002). The current results may not reflect the characteristics of stored representations of familiar objects but rather the bottom-up process of perceiving familiar objects. Determining orientation does not necessarily facilitate identification. Mitsumatsu and Yokosawa (2002) reported that previous knowledge of object orientation did not diminish view-dependency in identification of depth-rotated familiar objects in line drawings. For plane-rotated objects, the independence of the identification process from the orientation determi- nation process has also been demonstrated (De Caro \& Reeves, 2000; Harris \& Dux, 2005; Turnbull, Beschin, \& Della Sala, 1997). Logically, orientation-specific visual features are good cues for determining object orientation but useless for identification which should be performed independently of object orientation. Thus the mechanism of visual object perception may work using a trade-off between identification efficiency and orientation sensitivity. The possible relationships between the process of determining object orientation and the known characteristics of familiar object recognition, such as the viewpoint dependency of identification (e.g., Humphrey \& Jolicœur, 1993) and "canonical views" of familiar objects (Blanz, Tarr, \& Bülthoff, 1999; Palmer, Rosch, \& Chase, 1981), should be investigated further.

\section{AUTHOR NOTE}

This research was supported by a research fellowship of the Japan Society for the Promotion of Science (JSPS) for Young Scientists (awarded to R.N.) and by Grants-in-Aid for Scientific Research from the JSPS (awarded separately to R.N. and K.Y.). Correspondence concerning this article should be addressed to R. Niimi, Department of Psychology, Graduate School of Humanities and Sociology, University of Tokyo, 7-3-1 Hongo, Bunkyo-Ku, Tokyo 113-0033, Japan (e-mail: niimi@1 .u-tokyo.ac.jp).

\section{REFERENCES}

BARlow, H. B., \& ReEves, B. C. (1979). The versatility and absolute efficiency of detecting mirror symmetry in random dot displays. Vision Research, 19, 783-793.

Blanz, V., Tarr, M. J., \& Bülthoff, H. H. (1999). What object attributes determine canonical views? Perception, 28, 575-599.

Callahan, J., \& Weiss, R. (1985). A model for describing surface shape. In Proceedings of IEEE Conference on Computer Vision and Pattern Recognition (pp. 240-245). New York: IEEE Press.

De Caro, S. A., \& ReEves, A. (2000). Rotating objects to determine orientation, not identity: Evidence from a backward-masking/dual-task procedure. Perception \& Psychophysics, 62, 1356-1366.

HARRIS, I. M., \& DUX, P. E. (2005). Orientation-invariant object recognition: Evidence from repetition blindness. Cognition, 95, 73-93.

HeEley, D. W., \& Timney, B. (1988). Meridional anisotropies of orientation discrimination for sine wave gratings. Vision Research, 28, 337-344.

Humphrey, G. K., \& Jolicceur, P. (1993). An examination of the effects of axis foreshortening, monocular depth cues, and visual field on object identification. Quarterly Journal of Experimental Psychology, 46A, 137-159.

KoEnderink, J. J., \& VAn DooRn, A. J. (1979). The internal representation of solid shape with respect to vision. Biological Cybernetics, 32, 211-216.

Lawson, R., Humphreys, G. W., \& Joliceur, P. (2000). The combined effects of plane disorientation and foreshortening on picture naming: One manipulation or two? Journal of Experimental Psychology: Human Perception \& Performance, 26, 568-581.

McBeath, M. K., Schiano, D. J., \& Tversky, B. (1997). Threedimensional bilateral symmetry bias in judgments of figural identity and orientation. Psychological Science, 8, 217-223.

Mitsumatsu, H., \& Yokosawa, K. (2002). How do the internal details of the object contribute to recognition? Perception, 31, 1289-1298.

Palmer, S., Rosch, E., \& Chase, P. (1981). Canonical perspective and the perception of objects. In J. Long \& A. Baddeley (Eds.), Attention and performance $I X$ (pp. 135-151). Hillsdale, NJ: Erlbaum.

Sakata, H., Taira, M., Kusunoki, M., Murata, A., Tsutsui, K., TANAKA, Y., ET AL. (1999). Neural representation of three-dimensional features of manipulation objects with stereopsis. Experimental Brain Research, 128, 160-169.

TARR, M. J. (1995). Rotating objects to recognize them: A case study 
on the role of viewpoint dependency in the recognition of threedimensional objects. Psychonomic Bulletin \& Review, 2, 55-82.

Tarr, M. J., \& Kriegman, D. J. (2001). What defines a view? Vision Research, 41, 1981-2004.

Turnbull, O. H., Beschin, N. A., \& Della Sala, S. (1997). Agnosia for object orientation: Implications for theories of object recognition. Neuropsychologia, 35, 153-163.

Wagemans, J. (1995). Detection of visual symmetries. Spatial Vision, 9, 9-32.

WANG, G., TANifuJi, M., \& TANAKA, K. (1998). Functional architecture in monkey inferotemporal cortex revealed by in vivo optical imaging. Neuroscience Research, 32, 33-46.

Zabrodsky, H., Peleg, S., \& Avnir, D. (1995). Symmetry as a con- tinuous feature. IEEE Transactions on Pattern Analysis \& Machine Intelligence, 17, 1154-1166.

\section{NOTE}

1. Baseball helmet, camera, copying machine, desk, desk lamp, grand piano, house, kyusu (Japanese teapot), public telephone, and register. Similar to the symmetric stimuli objects (Figure 2C), they all have a salient front-back axis and lack a salient axis of elongation.

(Manuscript received March 6, 2006; revision accepted for publication April 9, 2007.) 